\title{
Training surgeons and the informed consent discussion in paediatric patients: a qualitative study examining trainee participation disclosure
}

\author{
Kunal Bhanot, ${ }^{1,2}$ Justin Chang, ${ }^{\oplus 1,2}$ Samuel Grant, ${ }^{1,2}$ Annie Fecteau, ${ }^{3}$ Mark Camp ${ }^{1,2}$
}

To cite: Bhanot K, Chang J, Grant S, et al. Training surgeons and the informed consent discussion in paediatric patients: a qualitative study examining trainee participation disclosure. BMJ Open Quality 2019;8:e000559. doi:10.1136/ bmjoq-2018-000559

- Additional material is published online only. To view please visit the journal online (http://dx.doi.org/10.1136/ bmjoq-2018-000559).

Received 23 October 2018 Revised 19 April 2019 Accepted 29 April 2019

\section{Check for updates}

(c) Author(s) (or their employer(s)) 2019. Re-use permitted under CC BY-NC. No commercial re-use. See rights and permissions. Published by BMJ.

${ }^{1}$ Division of Orthopaedic Surgery University of Toronto, Toronto,

Ontario, Canada

${ }^{2}$ Division of Orthopaedic Surgery, Hospital for Sick Children,

Toronto, Ontario, Canada

${ }^{3}$ Division of General and

Thoracic Surgery, Hospital for

Sick Children, Toronto, Ontario, Canada

Correspondence to

Dr Justin Chang;

justin.chang@mail.utoronto.ca

\section{ABSTRACT}

Background The process of obtaining informed consent is an important and complex pursuit, especially within a paediatric setting. Medical governing bodies have stated that the role of the trainee surgeon must be explained to patients and their families during the consent process. Despite this, attitudes and practices of surgeons and their trainees regarding disclosure of the trainee's participation during the consent process has not been reported in the paediatric setting.

Methods Nineteen face-to-face interviews were conducted with surgical trainees and staff surgeons at a tertiary-level paediatric hospital in Toronto, Canada. These were transcribed and subsequently thematically coded by three reviewers.

Results Five main themes were identified from the interviews. (1) Surgeons do not consistently disclose the role of surgical trainees to parents. (2) Surgical trainees are purposefully vague in disclosing their role during the consent discussion without being misleading. (3) Surgeons and surgical trainees believe parents do not fully understand the specific role of surgical trainees. (4) Graduated responsibility is an important aspect of training surgeons. (5) Surgeons feel a responsibility towards both their patients and their trainees. Surgeons do not explicitly inform patients about trainees, believing there is a lack of understanding of the training process. Trainees believe families likely underestimate their role and keep information purposely vague to reduce anxiety.

Conclusion The majority of surgeons and surgical trainees do not voluntarily disclose the degree of trainee participation in surgery during the informed consent discussion with parents. An open and honest discussion should occur, allowing for parents to make an informed decision regarding their child's care. Further patient education regarding trainees' roles would help develop a more thorough and patient-centred informed consent process.

\section{INTRODUCTION}

Surgeon educators are tasked with balancing the dual responsibilities to their patients and to their trainees' education. Patient-centred care has increasingly become the goal for healthcare organisations around the world, described as focusing delivery of care on patient preferences and needs. ${ }^{1}$ Concerns have arisen about the effect this model of care may have on the training of future surgeons, and how surgeons deal with patient queries and preferences regarding trainee involvement in their care. ${ }^{2}$

Obtaining informed consent is an essential element of the patient-surgeon encounter. It is a means of delivering information and provides the surgeon with the opportunity to establish trust and rapport. The process of obtaining informed consent is a complex and much debated pursuit. Surgeons should respect the patient's autonomy by describing the risks and benefits of a procedure, as well as alternatives to the procedure, in sufficient detail to give patients the ability to make an informed decision. This process is further complicated in the paediatric population, where parents may be responsible for providing consent on behalf of their child.

Medical governing bodies have stated that the presence, stage of training and level of involvement of trainees must be disclosed. ${ }^{3}$ Consent forms for surgery commonly detail that trainees will play a role in operative procedures; however, how routinely this is discussed with parents of paediatric patients undergoing surgery during the consent discussion is not known.

The fundamental measure of informed consent is often referred to as the 'reasonable person standard'. ${ }^{4}$ The idea of what is a reasonable approach to informed consent in the surgeon-patient relationship is much debated. Studies have shown that very detailed information about the procedure and trainee involvement did not increase patient anxiety and helped inform health-related decisions. ${ }^{5}$

Clinicians also have an 'ethical and social obligation' to adequately mentor their trainees to meet the healthcare needs of the next generation. ${ }^{6}$ However, it is not clear how, and to what extent, an explanation of the role of trainees fits within the informed consent 
process. A recent report found that although patient concerns regarding trainee participation was uncommon, it occurred frequently enough to warrant consideration. ${ }^{7}$ This report suggested that clear guidelines should be developed to confirm patient consent to trainee involvement and facilitate better patient understanding of the role of trainees and how they are supervised.

It is clear from the literature that patients would prefer a greater level of information regarding the role of trainees in their operative care,${ }^{8-12}$ but specifics surrounding the content and depth have yet to be determined. Gan et al found that when scripted disclosure was used during the informed consent process to indicate that trainees would be involved, $95 \%$ of patients agreed to trainee participation in their operation. ${ }^{13}$ Firdouse $e t a l^{14}$ also found that parent anxiety did not increase when trainee participation was covered during the consent discussion. In contrast, Porta $e t a l^{8}$ found rates of willingness to consent declined significantly when it was disclosed that trainees would be acting as the operating surgeon. A decrease in consent rate was also seen as the level of trainee became more junior. They suggest a consequence of a more detailed disclosure regarding trainee participation could result in a decline in patients providing consent and impact surgical training.

The attitudes and practices of surgeons and their trainees regarding disclosure of the trainee's participation in surgery during the consent process have not been reported in the paediatric setting. Previous literature indicates that many surgeons do not believe it necessary to disclose the role of trainees when gaining informed consent. ${ }^{14}$ Further information is necessary regarding the depth to which surgeons and surgical trainees explain the participation of trainees when obtaining informed consent from patients, particularly within the paediatric setting, given the added complexity of parental involvement.

The purpose of our study was to discern the disclosures pertaining to trainee involvement during surgery made during the consent discussion. Specific goals of these interviews included exploring the current practices during consent disclosure and identifying barriers to explaining the role of trainees during consent discussions with parents.

\section{METHODS}

\section{Design}

We used qualitative analysis to identify trainee and surgeon-derived concepts and themes regarding the disclosure of trainees' roles during the informed consent process in accordance with grounded theory methodology. ${ }^{15} 16$

\section{Setting and participants}

Semi-structured, face-to-face interviews were conducted by surgical trainees. Participants included surgical trainees and surgeons at an academic children's hospital. Surgical trainees interviewed ranged from post graduation year 2 to 5 . Surgeons from a variety of specialties were interviewed, including urology, plastic surgery, general surgery and orthopaedic surgery. The hospital operates within a medical system that is funded by a single provincially-run medical insurance system. Interviews were conducted until theme and concept saturation was achieved. Saturation was achieved after 10 trainee and 12 surgeon interviews.

\section{Data collection and analysis}

A semi-structured interview guide (online supplementary appendix 1 and II) was used and iteratively altered, based on concepts and themes brought out in previous interviews. Interviews lasted 20-40 min. They were audio recorded using a speech recognition application, which automatically transcribed the speech to text. All transcriptions were then checked for accuracy by the interviewer immediately following the interview, secured and anonymised, and then verified by a second author (KB, JC, SG). Transcriptions were imported into MAXQDA V.12 software (Sozialforschung GmbH, Berlin, Germany) for analysis.

Qualitative content analysis was performed and themes were generated by assessing codes representing concepts and content from the interviews $(\mathrm{KB}, \mathrm{JC}) .{ }^{17}{ }^{18}$ Internal validity was enhanced using a constant comparative methodology and all interviews were assessed by three reviewers (KB, JC, SG).${ }^{19}$ The SRQR inventory was applied to ensure robustness of methodology.

\section{Ethical considerations}

These interviews were conducted as part of a Quality Improvement project approved by our institution and we obtained informed consent from each participant. To ensure confidentiality and privacy, audiotapes were destroyed after transcription. Interview transcripts were saved on a password-protected and encrypted computer after anonymisation.

\section{Patient and public involvement}

Semi-structured interviews were used to formulate themes based on participant responses. There were no patients involved in this study. Study participants will be informed of the results of this publication.

\section{RESULTS}

Thematic analyses of surgeon and trainee interviews revealed the following major themes.

\section{Surgeons do not consistently disclose the role of surgical trainees to parents}

Many surgeons report not explicitly volunteering the role of surgical trainees in procedures during consent discussions with parents. One common explanation is the time constraints in a busy clinical setting. 
There's just so much to get through on the consent that it's been difficult for me to figure out exactly how to explain it to parents.

Surgeons also believed that the final clinical outcome is the most important factor, and specific details about the surgery were less important.

Most of my time is spent assuring them what to expect in terms of the final outcome not so much the actual journey.

In addition to concerns of overwhelming parents with information, surgeons also acknowledge the challenges in explaining the specific roles of trainees.

I think one of the difficulties will be trying to convey to families the medical education system, what a trainee actually does and what one can expect from them.

If parents explicitly asked about the role a trainee would play, surgeons reported being honest about their involvement, although some qualified their response by stating how care is delivered at a teaching hospital. Their responses are often vague about a trainee's involvement, not delving into specifics, but rather indicating that a team is involved in their child's care. However, a few surgeons reported a recent change in their practice by now indicating what roles trainees play during a procedure.

I often use the analogy that we are flying an airplane. I'm the pilot but in the cockpit there will be a navigator and there will be a copilot. Maybe the copilot is the one that's got their hands on the controls while the pilot is stepping back or maybe there's times where that role is reversed.

Interestingly, some surgeons reported that they do not disclose trainee involvement because that information is provided in the hospital consent form, though they acknowledged that the form is not often read thoroughly by parents.

... No, I don't believe disclosure [of trainee involvement] is adequately covered in the informed consent form, it does mention it though.

Additionally, by virtue of seeking care at a University hospital, some respondents believed parents are aware of the involvement of surgical trainees in their child's care, although not necessarily the trainee's specific role. Surgeons feel ultimately responsible for the outcomes of their patients and are willing to intervene if issues arise during surgery.

This is a teaching hospital and I will be present during the entire case, I will not do the entire surgery... if I'm not happy with what's happening, I will take over.
Surgical trainees are purposefully vague in disclosing their role during the consent discussion without being misleading The majority of surgical trainees are purposefully vague in disclosing their role during the surgical consent process. There is the belief that parents may not be able to fully appreciate how surgical training is delivered and that increased disclosure may result in additional anxiety.

I think that without surgical background one cannot understand the way things run an operating room and that it is difficult for patients to appreciate the way a team functions in the operating room.

In order to limit parental anxiety and concerns, surgical trainees often cited being part of a team under the supervision of a staff surgeon as sufficient disclosure to parents.

I basically tell the patients that I am one of the residents for my staff, and I will be assisting the team and my staff with the case. It's not much of an omission but rather vague purposefully because I don't think it adds any value to the patient's experience to know exactly what stuff I'm doing.

Even when asked directly about their potential role in surgery, trainees are non-specific. They often stated not knowing their specific role during a procedure as a limitation to disclosing their involvement during the consent discussion.

No one wants their child to be a training ground. When parents ask directly, generally I say [that] I usually help with the staff through the case. They've never asked me what I will specifically be doing.

I generally say that a team will be taking care of the patient and don't specifically highlight what role I'll be playing typically because I don't know entirely what my role will be until starting the procedure.

Many participants were concerned that complete disclosure of their roles and the extent of their involvement may result in curtailing of their surgical exposure and impact their training.

If they are given information about what my specific role will be, I find that then there may be limitations into how comfortable they are with proceeding with surgery at a teaching center.

This was a consistent concern for surgical trainees, who believe that informing parents of the specific roles of trainees during surgery would impact the consent process and be detrimental to their training.

I feel that if informing them [parents] limited the amount of surgical exposure I was receiving, then that would be detrimental to my training.

Supervising surgeons were also purposefully vague during the consenting process, for many of the reasons discussed above. 
I would never intentionally hide information from them if they asked about it but probably I'm not as explicit about some of the details.

\section{Surgeons and surgical trainees believe parents do not fully understand the specific role of surgical trainees}

Trainees believed their precise role and involvement in patient care is unclear to parents. They feel that parents are aware of them as being members of the team but their specific roles are not known, particularly their roles in the operating room. Many believe that by virtue of seeking care at an academic centre, parents expect and accept trainee involvement in their child's care.

I think most families coming to a teaching center realize that there are trainees in the room but I don't think that they are fully aware of the degree of involvement.

While parents appear to understand that trainees will be involved in the care of their child, it is believed that they do not appreciate the differences in surgical trainee levels of training.

I don't think they have a good understanding of doctors [at different stages of training] and what is done by trainees. I think if the staff would introduce a resident [surgical trainee] or fellow they would always use title of 'Dr.' but they would not differentiate the level training.

Importantly, the majority of trainees felt that there was a mismatch between parent perception of trainee involvement in the operating room and reality. It is thought that parents believe that the staff surgeon generally does the majority of the operation, with occasional assistance from trainees.

I do think that the patient's perception of who's doing the surgery and what are the important parts the surgery aren't necessarily the way things work in the operating room.

It is also thought that parents expect the staff surgeon to intently supervise trainees when given the opportunity to participate in steps of the operation. However, during a graduated responsibility model of training, often staff surgeons may not be directly participating in surgery. There is a consensus among trainees that if parents knew that staff surgeon was not always present during the operation, it would cause significant concern and anxiety.

I am sure they [the parents] would be concerned they knew the staff person [surgeon] was not in the room or in the hospital as his name is on the consent and they are the most responsible physician.

The fact that parents do not realise that the staff surgeon is not present in the operating room does not appear to be an ethical dilemma for trainees. They believe that the ultimate responsibility for the patient is with the staff surgeon even if they are not physically in the operating room.

And in being honest, I think that any person may be holding a knife, but the staff is the person who's in charge of surgery.

In addition, trainees believe that the final outcome is the most significant factor, and the exact degree of supervision is less important if the patient has a good outcome.

I think that the outcome of the surgeries that we perform is excellent. We know that staff will not allow us to do a poor job. If the procedure is not going well, they won't trust us to do a procedure. We have to maintain a high standard.

\section{Graduated responsibility is an important aspect of training surgeons}

A trainee's level of autonomy in the operating room is decided by several factors assessed by surgeons, including level of training, previous experience with the trainee, observed graduation of skills, prior training and the reputation of the trainee.

I observe them, and try to get an understanding of their level of skills. I need to be comfortable with them before I allow them to do more complex things in the OR.

While staff surgeons feel a responsibility to train their trainees, they do not believe that it should compromise patient outcomes.

I have trainees at the highest level of their training assisting me. There will be times when they're holding the knife but I'm there. I'm in charge and responsible for patient care.

Similarly, surgical trainees believe that their responsibility in the operating room increases gradually throughout their training.

When you're observed by your staff surgeon for smaller tasks and as you prove yourself, you get increasing amounts of autonomy.

As trainees progress through their education, they complete larger and more complex portions of the surgery. While surgical trainees welcome direct observation and teaching in the operating room, they appreciate the need for increased independence.

I think it's valuable to have them [surgeons] in the room as the ultimate responsibility does lie with them. However, at times the surgical experience for trainees is hindered by this.

They report this model of graduated responsibility to be extremely important for their training. Trainees understand that the staff surgeon is ultimately responsible for the patient and believe that their skills, level of training 
and previous experience with the surgeon determine the amount of independence a trainee will have.

I think that the staff surgeons should be responsible for them [patients]. However, I think that trainees do need to hone their skills by letting them be more independent.

It was noted that as trainees become more independent, there are times where the staff surgeon may not be present, though another more qualified trainee could be assisting.

Sometimes the staff [surgeons] are not in hospital but are available [to attend a surgery for which they are listed as the most responsible physician]. Sometimes they are in their office.... At times they may or may not even scrub for the surgery.

\section{Surgeons feel a responsibility towards both their patients and their trainees}

Surgeons often state that they bear the ultimate responsibility for their patients in terms of how the procedure is carried out.

Ultimately it's my responsibility if something bad happens.

Most surgeons report the importance of trust between them and the parents and often use the consent discussion to help build this.

I indicate that I am overall responsible and my contract with them is for me to be looking after their child.

During these discussions, parents would at times ask surgeons to perform the entire procedure. Most surgeons indicated that they do not change their practice despite receiving such requests and highlight the importance of the entire team being involved.

I may say to them 'well you know this is a teaching hospital unfortunately we have trainees that do take part, and if you aren't happy with that you can go elsewhere'.

Despite this, surgeons strive to balance the specific patient factors, parental requests and educational needs for trainees.

I've tried to strike a balance and try to tailor the amount of supervision to trainees, the patients and their educational level.

Surgeons also reported feeling a social responsibility to ensure competent surgeons are trained for the future.

We are responsible for teaching the next generation of surgeons to look after the next generation of patients.

\section{DISCUSSION}

Respect for patient autonomy is an essential part of the surgeon-patient relationship. Regard for this tenet is even more important when parents are required to make medical decisions for their children. Surgeons have a fiduciary duty towards their patients and must abide by legal and ethical obligations to disclose the risks, benefits and alternatives for a proposed procedure.

A critical component of the consent discussion is disclosure and identification of the surgeon performing the procedure. Although this is a relatively straightforward practice in non-teaching healthcare facilities, the extent to which trainee participation is disclosed is controversial. ${ }^{8}$ Previous reports have demonstrated patients are unaware of the involvement of medical students and residents in their care. ${ }^{20}$ We report the extent to which trainee participation in paediatric surgical procedures is disclosed to parents during the consent discussion. We also explore the attitudes of surgeons towards resident education and report on the perspectives of trainees during participation in these cases.

Through in-depth interviews, we were able to appreciate a thorough understanding of perspectives from both surgeons and trainees. Using this methodology, we were able to understand the motivations behind decisions made regarding disclosure pertaining to trainee involvement.

In keeping with reports of infrequent trainee participation disclosure in consent discussions for adult surgical procedures, our thematic analysis of interviews from both surgeons and trainees reveals similar findings in the paediatric setting where parents provide consent. ${ }^{21}$ These results were consistent among surgeons from a wide range of surgical specialties.

Surgeons and trainees endeavour to provide the best care possible to their patients. They aim not to be purposefully deceitful, but are vague when disclosing trainee participation. Trainees felt such disclosures were not made for fear of limiting their participation. This belief is supported by reports indicating the amount of trainee participation in procedures patients agreed to declined as the degree of involvement increased. ${ }^{821}$ Both trainees and surgeons noted that trainee involvement was listed as part of the consent form used by the hospital, but indicated parents often did not make note of this statement. When asked specifically by patients about trainee participation, both trainees and surgeons disclosed their involvement. However, the degree of involvement was not completely discussed. Not knowing what role a trainee will play until the time of surgery was also cited as a reason for not being able to completely disclose their participation. This suggests that increased patient education regarding the surgical training process may allow for a more open and informed discussion regarding trainee participation when obtaining consent.

Some surgeons reported concerns around parents not being able to grasp the complexities of the procedure during the consent discussion as a barrier to trainee 
participation disclosure. This paternalistic approach compromises patient autonomy and is not endorsed by regulatory bodies throughout the world. Statements from the American Medical Association, the American College of Surgeons and the Canadian Medical Protective Association mandate disclosure of trainee involvement. Consistent with previous reports, many surgeons believe that they bear the ultimate responsibility for the child and outcome; this may be a reason for non-disclosure. ${ }^{21}$ This sentiment was echoed by trainees as well who felt that in the end, the ultimate responsibility did rest with the surgeon-who was 'in charge'.

Both surgeons and trainees believe surgical training should involve graduated responsibility with increasing independence as skills and experience enhance. Surgeons indicate that trainee education is an important activity and that this must be balanced with patient care. Respect for trainees is an important component of their training and essential for their ability to participate in surgical procedures and provide care for the child throughout their stay in hospital. This increase in independence, however, does raise concerns for an increased incidence of the phenomenon of 'ghost' surgery, where the procedure is performed by someone other than the surgeon disclosed to the patient. ${ }^{22}$ Legal precedents in the USA were set with trainees deemed to have committed battery, and staff surgeons fraud, in cases where 'ghost' surgery was performed. ${ }^{23}$

Balancing a parent's need for a comprehensive consent discussion with the obligation to train the next generation of surgeons poses a great challenge. As patients do want to know about trainee involvement, we may presume that parents of children undertaking surgical procedures would wish for the same. ${ }^{8}$ In addition to the moral obligation of trainee participation disclosure, the College of Physicians and Surgeons of Ontario, the regulatory body in our jurisdiction, mandates that when a significant portion or all of a procedure is to be performed by a trainee, this must be disclosed to the patient. Given the lack of compliance with this policy as highlighted in our study, it behoves us to help educate both medical practitioners and patients. Disclosure of a trainee's role and model of graduated training, including how at times the majority of the procedure may be performed by a trainee, must be disclosed. A patient's ability to provide informed consent is stifled when such disclosures are not made as it may have a direct impact on their care.

Medical errors involving trainees were investigated using retrospective malpractice claims and reveal that nearly $27 \%$ of errors over a 22-year period were a direct consequence of trainee involvement with just over half of those errors being a result of technical incompetence. ${ }^{24}$ It should be noted that not all of these errors were in a surgical setting. This finding has direct implications for patient safety and once again highlights the importance of the informed consent discussion including trainee participation disclosure.
Transparency may help surmount this challenge through educational tools aimed at informing parents about the role trainees play in their child's care. As surgeons and trainees believe that it is difficult to discuss the precise steps a trainee may perform during a procedure, a general tool indicating their level of training in a teaching hospital environment would be beneficial. This quality improvement initiative would not be in place of the informed consent discussion, but rather an adjunct so that parents may better understand the role a trainee plays and their degree of involvement thereby providing a more informed consent. Such a tool may help mitigate the consent attrition rates reported by patients when trainee participation is disclosed.

\section{Limitations}

Thematic analysis of interviews from surgeons and trainees that practice in a single institution, in addition to variations in methods of surgical teaching across the world, may limit the generalisability of our findings. However, the involvement of trainees in surgical and medical patient care is ubiquitous. The informed consent discussion is undoubtedly always aimed at providing parents and patients with the best possible information to inform their decisions. These conversations take place throughout the world and by highlighting and hopefully addressing the reasons why trainees and preceptors do not fully disclose how training is undertaken, we hope to help guide better and more thorough informed consent discussions aimed at improving patient safety and improving the quality of consent discussions.

\section{CONCLUSIONS}

Our findings suggest that most surgeons and surgical trainees do not voluntarily disclose the degree of trainee participation in surgery during the informed consent discussion with parents. These findings are consistent with reports from similar investigations carried out in adult care settings. It is challenging to balance the duty a surgeon has to their patient, or the parents of a patient, with the educational needs of the trainee. Transparency is paramount to maintaining trust in the patient-surgeon relationship and an open and honest discussion should occur, allowing for parents to make informed decisions. Development of a patient educational tool to help facilitate this may be beneficial.

Contributors KB was responsible for study design, data collection and manuscript preparation. JC was responsible for study design, data collection, manuscript preparation and submission. SG collected data and prepared the manuscript. AF designed the study and edited the manuscript. MC was responsible for study design, data collection, manuscript preparation and editing.

Funding The authors (KB, JC, SG, AF and MC) have no sources of funding to declare.

Competing interests Each author (KB, JC, SG, AF and MC) certifies that he or she has no commercial associations (eg, consultancies, stock ownership, patent/ licensing arrangements, etc) that might pose a conflict of interest in connection with the submitted article.

Patient consent for publication Not required. 
Ethics approval This study was approved by the Research Ethics Board through an expedited quality improvement initiative at The Hospital for Sick Children, Toronto, Canada.

Provenance and peer review Not commissioned; externally peer reviewed.

Data availability statement Data are available on reasonable request.

Open access This is an open access article distributed in accordance with the Creative Commons Attribution Non Commercial (CC BY-NC 4.0) license, which permits others to distribute, remix, adapt, build upon this work non-commercially, and license their derivative works on different terms, provided the original work is properly cited, appropriate credit is given, any changes made indicated, and the use is non-commercial. See: http://creativecommons.org/licenses/by-nc/4.0/.

\section{REFERENCES}

1. Luxford K, Safran DG, Delbanco T. Promoting patient-centered care: a qualitative study of facilitators and barriers in healthcare organizations with a reputation for improving the patient experience. Int J Qual Health Care 2011;23:510-5.

2. Reilly BM. Don't learn on me-are teaching hospitals patientcentered? N Engl J Med 2014;371:293-5.

3. College of Physicians and Surgeons of Ontario. Professional responsibilities in postgraduate medical education, 2013. Dialogue. Available: http://www.cpso.on.ca/policies-publications/policy/ professional-responsibilities-in-postgraduate-medi

4. McCullough LB, Jones JW, Brody BA. Surgical ethics. New York: Oxford University Press, 1998.

5. Kerrigan DD, Thevasagayam RS, Woods TO, et al. Who's afraid of informed consent? BMJ 1993;306:298-300.

6. Jones JW, McCullough LB. Consent for residents to perform surgery. J Vasc Surg 2002;36:655-6.

7. Counihan TC, Nye D, Wu JJ. Surgeons' experiences with patients' concerns regarding trainees. J Surg Educ 2015;72:974-8.

8. Porta CR, Sebesta JA, Brown TA, et al. Training surgeons and the informed consent process: routine disclosure of trainee participation and its effect on patient willingness and consent rates. Arch Surg 2012;147:57-62.

9. Barnes HV, Albanese M, Schroeder J. Informed consent: the use of inpatients as teaching-patients for sophomore medical students. $J$ Med Educ 1980;55:698-703.
10. Gaeta T, Torres R, Kotamraju R, et al. The need for emergency medicine resident training in informed consent for procedures. Acad Emerg Med 2007;14:785-9.

11. Nguyen T-N, Silver D, Arthurs B. Consent to cataract surgery performed by residents. Can J Ophthalmol 2005;40:34-7.

12. Wisner DM, Quillen DA, Benderson DM, et al. Patient attitudes toward resident involvement in cataract surgery. Arch Ophthalmol 2008;126:1235-9.

13. Gan KD, Rudnisky CJ, Weis E. Discussing resident participation in cataract surgery. Can J Ophthalmol 2009;44:651-4.

14. Firdouse $M$, Wajchendler $A$, Koyle $M$, et al. Checklist to improve informed consent process in pediatric surgery: a pilot study. $J$ Pediatr Surg 2017;52:859-63.

15. Camp M, Gross A, McKneally M. Patient views on financial relationships between surgeons and surgical device manufacturers. Can J Surg 2015;58:323-9.

16. Pope C, Mays N. Qualitative research: reaching the parts other methods cannot reach: an introduction to qualitative methods in health and health services research. BMJ 1995;311:42-5.

17. Sandelowski M. Whatever happened to qualitative description? Res Nurs Health 2000;23:334-40.

18. Milne JL, Moore KN. Factors impacting self-care for urinary incontinence. Urol Nurs 2006;26:41-51.

19. Carcary M. The research audit trial-enhancing trustworthiness in qualitative inquiry. Electronic Journal of Business Research Methods 2009;7:11-24.

20. Zener R, Wiseman D. Disclosure of the resident role in the interventional radiology suite: how do interventional radiologists balance patient care and resident education? Can Assoc Radiol J 2016;67:409-15.

21. Knifed E, Taylor B, Bernstein M. What surgeons tell their patients about the intraoperative role of residents: a qualitative study. $A m \mathrm{~J}$ Surg 2008;196:788-94.

22. McAlister $C$. Breaking the silence of the switch--increasing transparency about trainee participation in surgery. $N$ Engl $\mathrm{J}$ Med 2015;372:2477-9.

23. Kocher MS. Ghost surgery: the ethical and legal implications of who does the operation. J Bone Joint Surg Am 2002;84:148-50.

24. Singh H, Thomas EJ, Petersen LA, et al. Medical errors involving trainees: a study of closed malpractice claims from 5 insurers. Arch Intern Med 2007;167:2030-6. 